\title{
Extraction of Speech Parameters from Speech Database using Festival
}

\author{
Sangramsing N. Kayte \\ Department of Computer Science \& IT \\ Dr. Babasaheb Ambedkar Marathwada University, \\ Aurangabad.
}

\begin{abstract}
Speech synthesis is the process of production of artificial speech. The system used for generation of speech from text is called as text-to-speech (TTS) system. In TTS system, text and voice models for a particular language or multiple languages are given as input to the system, which generates speech as output corresponding to the provided voice models. Speech synthesis systems can be extremely useful to people who are visually challenged, visually impaired and illiterate to get into the mainstream society. More recent applications include spoken dialogue systems and communicative robots. HMM (Hidden Markov Model) based Speech synthesis is the emerging technology for TTS. HMM based speech synthesis system consists of training phase and synthesis phase. In the training part, phone and excitation parameters are extracted from speech database and modeled by context dependent HMMs. In synthesis part, the system will extract the suitable phone and excitation parameters from the previously trained models and generates the speech. The main objective of this project is to build an HMM based speech synthesis system. In the training process, the system uses HTK (Hidden Markov Model Tool Kit) and SPTK (Signal Processing Tool Kit) developed at Cambridge University and Tokyo Institute of Technology respectively. Synthesis part is be done by "Festivale. Festival is a speech synthesis tool for the generation of speech and it is language independent which is developed at the University of Edinburgh. The main advantage of this approach is its flexibility in changing speaker identities, emotions and speaking styles.
\end{abstract}

\section{Keywords}

HMM (Hidden Markov Model), SPTK, HTK.

\section{INTRODUCTION}

HTS system consists of training part and synthesis part. In the training part, speech parameters are extracted from the speech database [1]. As training part is the heart of the HTS system, speech database has an important role in the implementation or design of the system [2]. A few software installations were required in order to create a working environment for implementation of this system [3]. HTS is the back-end part of the system. It does not contain text normalization module. So, in order to generate the speech, the HMM models are given to the front-end part of the system [4].

This Research paper explains the process undertaken to develop a speech synthesis system using Hidden Markov Models (HMM) [5]. It gives the overall information about the process starting from the selection of sentences for reading and the recording process. Further, gives the software tools required for the development of HMM Based Speech Synthesis System and also discusses the step by step procedure for the implementation of the system.

\author{
Monica Mundada \\ Department of Computer Science \& IT \\ Dr. Babasaheb Ambedkar Marathwada University, \\ Aurangabad.
}

\section{SPEECH DATABASE}

In the development of HMM based speech synthesis system, the speech database is given to the training part of the system for the extraction of speech parameters. The speech database includes recorded wave files and corresponding utterances of the recorded wave files [6-8]. The sentences to be recorded are selected in a way such that the text should cover maximum all phone symbols belonging to that particular language. In this thesis, for the implementation of HTS system, an Indic-Marathi prompts are taken for recording which is prepared by the people of Speech Vision Lab.To record speech files for the chosen text, the speaker should be selected in such a way that he or she can speak with pleasant voice [9]. The speaker should not suffer from bad health condition like cold, cough etc. The selected speaker should have some awareness about the selected prompts to avoid mistakes in the recording process [10-25]. To record an efficient and noise-less speech database, high quality recording equipment is required. A noise less environment like a specially designed recording studio is required to avoid background noise while recording the speech files. Recording is done using a builtin laptop microphone. The speech files are recorded in noise-less clean environment. A high quality microphone and recorder are used for recording. The distance from the microphone to mouth, speaking volume and speaking style is kept constant till the completion of recording. A MATLAB program is used while recording for the generation of single wav files. At the starting and ending of sentence recording, a key is pressed such that the respective recorded speech file will be stored with the given name as a single wave file.Care should be taken to avoid mistakes in utterances due to wrong pronunciation or repeated pronunciation of a word while recording [11]. Any mistakes while recording can be recovered either by re-recording those utterances or by correcting the corresponding sentence to suit the sentence [12]. The recorded speech files are stored in wave format (.wav) [13-25].

\section{TOOLS}

Some software installations are required for the development of HMM based speech synthesis system. This HTS system was build on laptop with Ubuntu 14.04 as operating system on a 32-bit machine and can be build on any version with the supporting library files. These tools can be found at their respective sites [26-38].

\subsection{List of required tools}

The tools used for the implementation of HTS system are listed below:

- $\quad$ speech_tools-2.1-release.tar.gz

- festival-2.1.tar.gz

- festvox- 2.1-release.tar.gz

- HTK-3.4.1.tar.gz 
- Hdecode-3.4.1.tar.gz

- HTS-2.2_for HTK-3.4.1.tar.gz

- $\quad$ SPTK-3.4.1.tar.gz

- hts_engine_API-1.05.tar.gz

- ActiveTc18.4.19.6.295590-linux-ix86.tar.gz

- HTS-demo_CMU-ARCTIC-SLT.tar.bz2

\section{IMPLEMENTATION OF HTS SYSTEM}

Create a directory or folder on the desktop and move to that directory.

- mkdir directory_name

- cd directory_name

Copy all the required downloaded tools into the directory_name folder [4][5].

\subsection{Installation of speech tools, festival and festvox}

To build HMM models, the utterance files which consist of textual features and the duration of each unit in the text to be synthesized are required. To generate these utterance structures, speech tools, festival and festvox are needed. Run the commands given, for the installation of speech tools, festival and festvox respectively [4][5].

- Installation of speech tools

- $\quad \operatorname{tar}-\mathrm{xvf}$ speech_tools-2.1-release.tar.gz

- cd speech tools

- ./configure

- Make

The first command is for extraction tar files. The most popular archiving tool used in UNIX and Linux is the "tar" command. The second command gives the path to the speech files folder. Next command is for running the configure script. "Configure" is an executable script designed to help in developing a program to be run on computers and matches the libraries on the user's computer, with those required by the program, before compiling it from its source code. By running this command, it will check some details about the machine on which the software is going to be installed and creates the Makefile to be used in the next step. The last command make is a general purpose workflow program, usually used for compilation [4][5].

- If error occurs during the execution of make command, follow the given steps:

- In speech_tools/include/EST_Titerrator, at line number 212:7 and 292:7, insert this-> and save.

- In speech tools/include/EST_TNamedEnum.h, at line no 133:64, insert this-> and save.

- In speech_tools/base_class/EST_Tsimplematrix.cc, at line no 132:4, 130:11, and 101:4 insert this-> before set_values, just_resize.

- $\quad$ Add \#include <string.h> in the header file section as this program consists of memcpy function.

- Inspeech_tools/base_class/EST_Tsimplevector.cc, at line no 74:7, insert this-> before just_resize. Add \#inclue<string.h> in the header files section because this program consists of memset function.

- $\quad$ Run make

\section{Installation of Festival}

- cd.

- $\quad \operatorname{tar}-\mathrm{xvf}$ festival-2.1.tar.gz

- cd festival

- ./configure

- make

\section{Installation of Festvox}

- cd.

- $\quad \operatorname{tar}-\mathrm{xvf}$ festvox- 2.1-release.tar.gz

- cd festvox

- ./configure

- Make

\subsection{Installation of HMM Toolkit (HTK) and Patch for HTS}

HTK along with patch files provided for HTS are used to train contextindependent and context-dependent hidden Markov models. The procedure for installing these tools is given below:

- cd.

- $\quad \operatorname{tar}-\mathrm{xvf}$ HTK-3.4.1.tar.gz

- $\quad \operatorname{tar}-x v f$ Hdecode-3.4.1.tar.gz

- $\quad \operatorname{tar}-\mathrm{xvf}$ HTS-2.2_for HTK-3.4.1.tar.gz

(Copy all the extracted files into a folder and name it as hts_patch)

- $\quad$ cd htk

Run the command given below to include a patch file for HTS.

- $\quad$ patch - p1 -d . . ./hts_patch/HTS-2.2_for_HTK-

\subsection{PATCH}

The executable such as Hcopy, HList, HInit, will be compiled in /usr/local/HTS-2.2beta/bin

- $\quad . /$ configure

- make

- $\quad$ sudo make install

- $\quad$ sudo make hlmtools install-hlmtools

- $\quad$ sudo make hdecode install-hdecode

\subsection{Installation of HTS Engine}

This synthesize speech waveform from trained HMMs

- cd.

- $\quad \operatorname{tar}-\mathrm{xvf}$ hts_engine_API-1.05.tar.gz

- cd hts_engine_API-1.05

- ./configure

- make

- $\quad$ sudo make install

\subsection{Installation of SPTK (Signal}

Processing Toolkit)

The SPTK functions such as mgecp, $\mathrm{x} 2 \mathrm{x}$, lsp2lpc, etc will be compiled in /usr/local/SPTK/bin

- cd..

- $\quad \operatorname{tar}-x v f$ SPTK-3.4.1.tar.gz

- ./configure

- make

- $\quad$ sudo make install 


\subsection{Installation of Active Tcl}

Execute the following commands to install Active $\mathrm{Tcl}$ in /usr/local/Active Tcl-8.4/bin/

- $\quad c d$.

- $\quad \operatorname{tar}-x v f$ ActiveTcl8.4.19.6.295590-linux-ix86.tar.gz

- $\quad$ cd ActiveTcl8.4.19.6.295590-linux-ix86

- $\quad$ sudo ./install.sh

In the window that appears, type /usr/local/ActiveTcl-8.4

\section{GENERATION OF RAW FILES AND UTTERANCES}

i) Generation of raw files from the wav files using ch_wave

\$ESTDIR/bin/ch_wave -otype raw -F 16000 wav/\$i.wav -o directory_name/HTS-demo_CMU-ARCTIC-

SLT/data/raw/db_v_\$i.raw ii) Generation of utterance files

Perform the steps given to set the environment variables for speech tools, festival and festvox directories respectively. These variables point to the paths where speech tools, festival and festvox tools are located.

- $\quad$ export

ESTDIR=/home/user_name/Desktop/directory_

- name/speech_tools

- $\quad$ export FESTDIR=/home/user_name/Desktop/directory_

- $\quad$ name/ festival

- export FESTVOXDIR=/home/user_name/Desktop/

- directory_name/festvox

To start a new project, a new folder is to be created. The folder to be created has to follow a particular standard which is given below. Create a directory in ,directory_name"e folder. The name of the folder should be in the format as shown below:

- mkdir institutename_dictonarylanguage_speaker name_type

- (ex. bamu_sangram_indic)

- Now move to the created directory to make setup. For this process, run the commands given below:

- $\quad \mathrm{Cd}$ institutename_dictonarylanguage_speakername_typ e

- $\quad$ \$FESTVOXDIR/src/clustergen/setup_cg

bamu sangram indic

After execution of the commands, required folders will be created and required shell script files will be copied in to the newly created directory. Next, we need to generate prompt file corresponding to the recorded speech wave files. It consists of file id followed by transcription. Transcription is quoted with double quotes and entire line is enclosed in brackets. As an example, the structure of prompt file is shown.

( mar0001 " kaarand a aapalayaakad:ei tii padadhata naahii." )

The prompt file is copied into the etc folder in the current directory for the input wave files and it is renamed as txt.done.data. Next, copy the recorded speech files which are in the .wav format into the recording folder which is already created in the current directory. These wav files which are in the recording folder may not be compatible for labeling. So, these wav files must be modified into the required format. For this modification, a script file exists in bin folder in current directory. To execute this file script, run the command which is given below:

\section{./bin/get_wavs recording/*.wav}

Wave files are extracted one by one from the recording folder and given to the ch_wave executable file which is part of the speech tools in exported environment variable. This executable file takes the wav as input and converts the wav file to required format; here the scripts require a wav file with $16000 \mathrm{HZ}$ sampling frequency and mono sound. The output wave is stored in wav folder which is in the current directory. Next, prompt files are to be generated for each and every speech file present in wav folder using txt.done.data prompt file which is in etc folder. This can be done by using script called do_build

\section{./bin/do_build build_prompts}

This generates prompt-wav files, prompt-lab files and promptutts files in existing folders. Now, the directory is ready to make labeling. The labeling is done by using EHMM labeler. An EHMM labeler is designed by festvox developing group. An EHMM labeler is available with festvox 2.1 release. The labeling procedure can be done by executing the command given below [4] [5].

\section{./bin/do_build label}

if error occurs during execution of this command, change the directory path at 148th line in do_ehmm script. (\$FESTVOXDIR/src/ehmm/bin/do_ehmm) Here, the phones present in speech files are labeled and these labels are stored in lab folder. These generated labels are used to create utterances for speech files. Run the below command for the generation of utterances[4] [5].

\section{./bin/do_build build_utts}

The generated utterance files are in the format that can be used by the HTSdemo_CMU-ARCTIC-SLT and these utterances are stored in folder named utts which is in the festival directory.

\subsection{Installation of HTS-demo_CMU- ARCTIC-SLT}

The HTS-demo is unpacked using the tar command as given below:

- $\quad c d$.

- $\quad$ tar -xvf HTS-demo_CMU-ARCTIC-SLT.tar.bz2

Delete all files on the paths data/raw and data/utts. Next, copy the raw files and utterances which are generated in the above steps into the data/raw and data/utts folders. While copying, it should be noted that the raw files and utterances should be in the format given below respectively.

Raw files: db_v_mar0001.raw

Utterances: db_v_mar0001.utt

Where ' $d b$ ' refers to the data set and ' $v$ ' refers to the speaker name.

All the necessary files are in the right places in their respective folders. The first stage in running the HTS-demo scripts is to configure it. Configuring the HTS-demo gives a guideline on how to set up these paths and alter certain parameters. It also indicates the packages required to run the HTS-demo. The command used to configure is given below: 


\section{- $\quad$ cd HTS-demo_CMU-ARCTIC-SLT}

Setup HTS-demo_CMU-ARCTIC-SLT by running configure script given below:

./configure--with-tcl-search-path=usr/local/ActiveTcl8.4/bin--with-fest-search- path=/home/

idntyti/Desktop/HTS/festival/examples--withsptk-searchpath=/usr/local/SPTK/bin--with-hts-

searchpath=/usr/local/HTS-2.2beta/bin--with-hts-enginesearchpath=/home/idntyti/Desktop /HTS/hts_engine_API$1.05 /$ bin

There are certain files which need to be modified in order to run the demo successfully. A slight modification is to be done in the file Makefile found in the

/data/ which is in the main HTS-demo_CMU-ARCTIC-SLT directory.

makefile \#setting

SPEAKER $=\mathrm{v}$

\section{DATASET $=\mathrm{db}$}

This modification depends on how the raw file (db_v_mar0001.raw) and utterance (db_v_mar0001.utt) are named. The speech parameters have to be changed according to recorded database in the Makefile, which is in the data folder in the

HTS-demo_CMU-ARCTIC-SLT/data/Makefile as given below: \# speech analysis conditions

SAMPFREQ $=16000$ \# Sampling frequency $(48 \mathrm{kHz})$

FRAMELEN $=400$ \# Frame length in point $(1200=48000$ $* 0.025)$

FRAMESHIFT $=80 \#$ Frame shift in point $(240=48000 *$ $0.005)$

Perform make command as given below with which HTSdemo runs: - make

With this make command; the script in the Makefile is executed and all the speech parameters required are extracted from the raw files.

Step by step execution procedure of Makefile:

- Extraction of mel generalized cepstral coefficients (mgc) from raw files. These coefficients are stored in HTS-demo_CMU-ARCTIC-SLT/data/mgc.

- Extraction of log-fundamental frequencies (If0) from raw files. These parameters are stored in HTSdemo_CMU-ARCTIC-SLT/data/lfO.

- Generation of training data files from mgc and lf0 files. These files are stored in HTS-demo_CMUARCTIC-SLT/data/cmp.

- Extraction of monophone and full context labels from utterance files. Monophone

- labels are stored in HTS-demo_CMU-ARCTICSLT/data/labels/mono and full context labels are formed in HTS-demo_CMU-ARCTICSLT/data/labels/full.

- Generation of full context model list file. This file is stored in HTS-demo_CMU-ARCTICSLT/data/lists/full.list.

- Generation of full context model list file which includes unseen models and this file is in HTSdemo_CMU-ARCTIC-SLT/data/lists/full_all.list.
- Generation of monophone list file. This file is stored in HTS-demo_CMU- ARCTICSLT/data/lists/mono.list.

Generation of training data script and generation label script. These scripts are

- stored in HTS-demo_CMU-ARCTICSLT/data/scp/train.scp and HTS-demo_CMUARCTIC-SLT/data/scp/gen.scp respectively.

Once the features and the context-dependent label files are obtained, contextdependent models are built and speech is synthesized by the following command. Config.pm contains a list of parameters such as the stream weights, feature order, etc., along with the model structure, file locations, and HTS commands and options.

- $\quad$ perl scripts/Training.pl scripts/Config.pm

With the execution of this command, in order to synthesize, the necessary files are formed in their respective folders.

Step by step execution procedure of Training.pl script is given below:

- Creation of required directories namely configs, models, stats, edfiles, trees, gv, voices, gen and proto in HTS-demo_CMUARCTIC-SLT.

- Generation of set of configuration files used by different modules in the HTS-demo_CMUARCTIC-SLT/configs.

- Creation of prototype of an HMM. The prototype is created in HTS-demo_CMU-ARCTIC-SLT/proto. Monophone (context-independent) models for each phone in the database are to be trained.

The synthesized wave files can be played using the play command. The waveform can be viewed using wave surfer. The properties of the synthesized speech can be viewed by using ch_wave. The models generated in the voice folder are used to generate speech for a given text when these models are provided to the front-end part of the synthesis system.

\section{GENERATION OF SPEECH WITH HMM MODELS FOR A GIVEN TEXT}

HTS is the backend of the synthesis system. Using only the HTS-demo scripts, speech cannot be synthesized from the given text. We need to plug HTS to a front-end. Festival can be used as front-end. Download the tar files from the link given below:

http://homepages.inf.ed.ac.uk/jyamagis/release/MarathiHTSV oices-ver1.0.tar.gz

Copy the downloaded tar file into the directory_name folder. Follow the given commands to extract the downloaded tar file.

- cd..

- $\quad \operatorname{tar}-\mathrm{xvf}$ MarathiHTSVoices-ver1.0.tar.gz

In the folder named MarathiHTSVoices, replace the models in the hts_voice_cmu_us_arctic_slt-1.03.tar.gz and hts_voice_cmu_us_arctic_slt-1.03.tar.gz with the models generated in the voice folder. Next, run the below commands for the extraction of tar files present in the folder.

- cd MarathiHTSVoices

- $\quad$ sh do_build 
After execution of this command, a new folder "build" is formed which contains the extracted files. A text document named "example.txt" exists in MarathiHTSVoices folder for the purpose of giving text as input. So, the text input is given through this document and have to save it. Replace the sampling frequency in runvoice.sh file by 16000 and frame rate by 80 . After that, run the command given below:

\section{- $\quad . /$ runvoice.sh}

After execution of this command, wave files are generated in the MarathiHTSVoices folder. Finally, the synthesized waveforms can be played by using the play command.

\section{CONCLUSION}

The process undertaken to develop a speech synthesis system using hidden Markov models is explained in detail. The overall information about the process, starting from the selection of sentences is given clearly in this chapter. At last, the procedure for implementation of HTS system is described in step by step procedure.

\section{REFERENCES}

[1] Sangramsing Kayte, Monica Mundada, Dr. Charansing Kayte "A Marathi Hidden-Markov Model Based Speech Synthesis System" IOSR Journal of VLSI and Signal Processing (IOSR-JVSP) Volume 5, Issue 6, Ver. I (Nov -Dec. 2015), PP 34-39e-ISSN: 2319 -4200, p-ISSN No. : $2319-4197$

[2] Sangramsing Kayte, Monica Mundada, Dr. Charansing Kayte "Implementation of Marathi Language Speech Databases for Large Dictionary" IOSR Journal of VLSI and Signal Processing (IOSR-JVSP) Volume 5, Issue 6, Ver. I (Nov -Dec. 2015), PP 40-45e-ISSN: 2319 -4200, p-ISSN No. : $2319-4197$

[3] Sangramsing Kayte, Dr. Bharti Gawali "Marathi Speech Synthesis: A review" International Journal on Recent and Innovation Trends in Computing and Communication ISSN: 2321-8169 Volume: 3 Issue: 63708 - 3711

[4] Sangramsing Kayte, Monica Mundada,Dr. Charansing Kayte" Speech Synthesis System for Marathi Accent using FESTVOX" International Journal of Computer Applications (0975 - 8887) Volume 130 - No.6, November 2015

[5] Sangramsing Kayte, Monica Mundada,Dr. Charansing Kayte "Screen Readers for Linux and Windows Concatenation Methods and Unit Selection based Marathi Text to Speech System" International Journal of Computer Applications (0975 - 8887) Volume 130 No.14, November 2015

[6] Monica Mundada, Bharti Gawali, Sangramsing Kayte "Recognition and classification of speech and its related fluency disorders" International Journal of Computer Science and Information Technologies (IJCSIT)

[7] Monica Mundada, Sangramsing Kayte, Dr. Bharti Gawali "Classification of Fluent and Dysfluent Speech Using KNN Classifier" International Journal of Advanced Research in Computer Science and Software Engineering Volume 4, Issue 9, September 2014

[8] Monica Mundada, Sangramsing Kayte "Classification of speech and its related fluency disorders Using KNN" ISSN2231-0096 Volume-4 Number-3 Sept 2014
[9] Sangramsing Kayte, Monica Mundada "Study of Marathi Phones for Synthesis of Marathi Speech from Text" International Journal of Emerging Research in Management \&Technology ISSN: 2278-9359 (Volume4, Issue-10) October 2015

[10] Sangramsing N.kayte "Marathi Isolated-Word Automatic Speech Recognition System based on Vector Quantization (VQ) approach" 101th Indian Science Congress Jammu University 03th Feb to 07 Feb 2014.

[11] Sangramsing Kayte, Monica Mundada, Bharti Gawali "PERFORMANCE EVALUATION OF SPEECH SYNTHESIS TECHNIQUES FOR ENGLISH LANGUAGE " International Congress on Information and Communication Technology 9-10 October, 2015

[12] Sangramsing Kayte, Monica Mundada, Dr. Charansing Kayte " Performance Calculation of Speech Synthesis Methods for Hindi language IOSR Journal of VLSI and Signal Processing (IOSR-JVSP) Volume 5, Issue 6, Ver. I (Nov -Dec. 2015), PP 13-19e-ISSN: 2319 -4200, pISSN No. : $2319-4197$

[13] Sangramsing Kayte, Monica Mundada,Dr. Charansing Kayte " Performance Evaluation of Speech Synthesis Techniques for Marathi Language " International Journal of Computer Applications (0975 - 8887) Volume 130 - No.3, November 2015

[14] Sangramsing Kayte,Dr. Charansing Kayte "Di-phoneBased Concatenative Speech Synthesis System for Hindi" International Journal of Advanced Research in Computer Science and Software Engineering -Volume 5, Issue 10, October-2015

[15] Sangramsing Kayte,Dr. Charansing Kayte "A Review of Unit Selection Speech Synthesis" International Journal of Advanced Research in Computer Science and Software Engineering -Volume 5, Issue 10, October-2015

[16] Sangramsing Kayte,Dr. Charansing Kayte "Di-phoneBased Concatenative Speech Synthesis Systems for Marathi Language" OSR Journal of VLSI and Signal Processing (IOSR-JVSP) Volume 5, Issue 5, Ver. I (Sep -Oct. 2015), PP 76-81e-ISSN: 2319 -4200, p-ISSN No. : $2319-4197$

[17] )Sangramsing Kayte,Dr. Charansing Kayte "A CorpusBased Concatenative Speech Synthesis System for Marathi" IOSR Journal of VLSI and Signal Processing (IOSR-JVSP) Volume 5, Issue 6, Ver. I (Nov -Dec. 2015), PP 20-26e-ISSN: 2319 -4200, p-ISSN No. : 2319 $-4197$

[18] )Sangramsing Kayte,Dr. Charansing Kayte "Marathi Text-To-Speech Synthesis using Natural Language Processing "IOSR Journal of VLSI and Signal Processing (IOSR-JVSP)Volume 5, Issue 6, Ver. I (Nov -Dec. 2015), PP 63-67e-ISSN: 2319 - 4200, p-ISSN No. : $2319-4197$

[19] Sangramsing Kayte,Dr. Charansing Kayte "Screen Readers for Linux and Windows - Concatenation Methods and Unit Selection based Marathi Text to Speech System" International Journal of Computer Applications (0975 - 8887) Volume 130 - No.14, November 2015

[20] Sangramsing Kayte," Hidden Markov Model based Speech Synthesis: A Review" International Journal of 
Computer Applications (0975 - 8887) Volume 130 No.3, November 2015

[21] Sangramsing N. Kayte,Dr. Charansing N. Kayte, Dr.Bharti Gawali "Approach To Build A Marathi Text-To-Speech System Using Concatenative Synthesis Method With The Syllable" Sangramsing Kayte et al.Int. Journal of Engineering Research and Applications ISSN: 2248-9622, Vol. 5, Issue 11, (Part-4) November 2015, pp.93-97

[22] Sangramsing N. Kayte, Dr. Charansing N. Kayte,Dr.Bharti Gawali* "Grapheme-To-Phoneme Tools for the Marathi Speech Synthesis" Sangramsing Kayte et al.Int. Journal of Engineering Research and Applications ISSN: 2248-9622, Vol. 5, Issue 11, (Part -4) November 2015, pp.86-92

[23] Sangramsing Kayte "Duration for Classification and Regression Tree for Marathi Text-to-Speech Synthesis System" Sangramsing Kayte Int. Journal of Engineering Research and Applications ISSN: 2248-9622, Vol. 5, Issue 11, (Part-4)November2015

[24] Sangramsing Kayte "Transformation of feelings using pitch parameter for Marathi speech" Sangramsing Kayte Int. Journal of Engineering Research and Applications ISSN: 2248-9622, Vol. 5, Issue 11, (Part -4) November 2015, pp.120-124

[25] Sangramsing Kayte,Dr. Charansing N. Kayte, Dr.Bharti Gawali " Artificially Generatedof Concatenative Syllable based Text to Speech Synthesis System for Marathi" OSR Journal of VLSI and Signal Processing (IOSRJVSP) Volume 5, Issue 6, Ver. II (Nov -Dec. 2015), PP 44-49e-ISSN: $2319-4200$, p-ISSN No. : $2319-4197$

[26] Sangramsing Kayte,Dr. Charansing N. Kayte, Dr.Bharti Gawali " Automatic Generation of Compound Word Lexicon for Marathi Speech Synthesis" OSR Journal of VLSI and Signal Processing (IOSR-JVSP) Volume 5, Issue 6, Ver.II (Nov -Dec. 2015), PP 25-30e-ISSN: 2319 -4200, p-ISSN No. : $2319-4197$

[27] Sangramsing Kayte,Dr. Charansing N. Kayte, Dr.Bharti Gawali "Approach of Syllable Based Unit Selection Text-To-Speech Synthesis System for Marathi Using Three Level Fall Back Technique OSR Journal of VLSI and Signal Processing (IOSR-JVSP) Volume 5, Issue 6, Ver. II (Nov -Dec. 2015), PP 31-35e-ISSN: $2319-4200$, p-ISSN No. : $2319-4197$

[28] Sangramsing Kayte,Dr. Charansing N. Kayte, Dr.Bharti Gawali "Implementation of Text To Speech for Marathi Language Using Transcriptions Concept" Sangramsing N. Kayte et al. Int. Journal of Engineering Research and Applications ISSN: 2248-9622, Vol. 5, Issue 11, (Part - 5) November 2015, pp.33-36

[29] Sangramsing Kayte,Dr. Charansing N. Kayte, Dr.Bharti Gawali "Rule-based Prosody Calculation for
Marathi Text-to-Speech Synthesis" Sangramsing N. Kayte et al. Int. Journal of Engineering Research and Applications ISSN: 2248-9622, Vol. 5, Issue 11, (Part 5) November 2015, pp.33-36

[30] Sangramsing Kayte, Dr.Bharti Gawali "The Marathi Text-To-Speech Synthesizer Based On Artificial Neural Networks " International Research Journal of Engineering and Technology (IRJET) e-ISSN: 23950056Volume: 02 Issue: 08| Nov-2015-ISSN: 2395-0072

[31] Sangramsing N. Kayte, Dr.Charansing N. Kayte, Dr.Bharti Gawali "The Heart of Generating Hidden Markov Model Which Are Essential For Synthesis" Journal Of Modern Engineering Research (IJMER) International OPEN ACCESS|ISSN: 2249-6645 Vol. 5 | Iss.12| December 2015 | 26.

[32] Sangramsing N. Kayte, Dr.Charansing N. Kayte, Dr.Bharti Gawali "The Prosody Subsystem and Pitch Pattern for Marathi Text To Speech Synthesis International Journal Of Modern Engineering Research (IJMER) OPEN ACCESS| ISSN: 2249-6645 |Vol. 5 | Iss. 12| December 2015 | 26

[33] Sangramsing Nathusing Kayte. "Festival and Festvox Framework Tools for Marathi Text-to-Speech Synthesis" International Journal of Computer Applications 132(4):38-43, December 2015. Published by Foundation of Computer Science (FCS), NY, USA

[34] Sangramsing Nathusing Kayte. Text To Speech for Marathi Language using Transcriptions Theory. International Journal of Computer Applications 131(6):39-41, December 2015. Published by Foundation of Computer Science (FCS), NY, USA

[35] Sangramsing Kayte, Bharti Gawali "A Text-To-Speech Synthesis for Marathi Language using Festival and Festvox" International Journal of Computer Applications (0975 - 8887)Volume 132 - No.3, December2015

[36] Sangramsing N Kayte, Charansing N Kayte and Bharti W Gawali. Article: Marathi Synthetic Voice using Synthesizer Modules of Festival Speech and HTS Straight Processing. Communications on Applied Electronics 3(7):9-12, December 2015. Published by Foundation of Computer Science (FCS), NY, USA

[37] Sangramsing Kayte "Marathi Speech Recognition System Using Hidden Markov Model Toolkit" International OPEN ACCESS Journal Of Modern Engineering Research (IJMER) | IJMER | ISSN: 22496645 || Vol. 5 | Iss. 12 | December 2015|13

[38] Sangramsing N.Kayte , Monica Mundada "Overview of Hidden Markov Model for Test-To-Speech Synthesis Methods " International Journal Of Engineering And Computer Science ISSN:2319-7242Volume -4 Issue -12 December, 2015Page No.15303-15311 